Research Article

\title{
Corrected Hawking Radiation of Dirac Particles from a General Static Riemann Black Hole
}

\author{
Ge-Rui Chen and Yong-Chang Huang \\ Institute of Theoretical Physics, Beijing University of Technology, Beijing 100124, China \\ Correspondence should be addressed to Ge-Rui Chen; chengerui@163.com
}

Received 7 April 2013; Revised 6 May 2013; Accepted 20 May 2013

Academic Editor: George Siopsis

Copyright (c) 2013 G.-R. Chen and Y.-C. Huang. This is an open access article distributed under the Creative Commons Attribution License, which permits unrestricted use, distribution, and reproduction in any medium, provided the original work is properly cited.

Considering energy conservation and the back reaction of radiating particles to the spacetime, we investigate the massive Dirac particles' Hawking radiation from a general static Riemann black hole using improved Damour-Ruffini method. A direct consequence is that the radiation spectrum is not strictly thermal. The correction to the thermal spectrum is consistent with an underlying unitary quantum theory and this may have profound implications for the black hole information loss paradox.

\section{Introduction}

In the 1970s, Hawking's astounding discovery that black holes radiate black body spectrum $[1,2]$ had greatly stimulated the development of the theory of black hole thermodynamics and then four laws of black hole thermodynamics were established $[3,4]$. Hawking radiation gives us new insights into gravity physics and also provides some hints of quantum gravity, so there have been a lot of works related to Hawking radiation until recently. Our concern here is about information conservation during Hawking radiation. From Hawking's famous work, people know that black holes are not the final state of stars, and, with the emission of Hawking radiation, they could lose energy, shrink, and eventually evaporate completely. However, because of the quality of purely thermal spectrum, it also sets up a disturbing and difficult information loss problem: what happens to information during black hole evaporation? And it also implies the loss of unitarity or, to put it more severely, the breakdown of quantum mechanics [5-9]. About the year of 2000, Parikh and Wilczek, contemplating Hawking's heuristic picture of tunneling triggered by vacuum fluctuations near the horizon, proposed a semiclassical method to investigate the emission rate by treating Hawking radiation as a tunneling process [10-12]. They found that the barrier of tunneling is created by the outgoing particle itself, and when energy conservation is considered, a corrected spectrum is given, which supports the underlying unitary theory. Subsequently, some references extended this method to more general circumstances [13-17]. All of the results obtain the conclusions that the spectrum is no longer precisely thermal and information can be taken out of the black hole, which means a possible explanation for information puzzle and the loss of quantum unitarity.

Considering the crucial points of energy conservation and the back reaction of particles to the spacetime in Parikh and Wilczek's method, [18-20] presented another method on the base of classical Damour-Ruffini's method to calculate modified Hawking radiation from the BTZ black hole and Kerr-Newman black hole and got the same result as Parikh and Wilczek's tunneling method. In this paper, we will use this method to calculate Dirac particles' Hawking radiation from the general Riemann black hole, and we obtain the same corrected Hawking radiation spectrum as the previous literature.

The remainder of this paper is organized as follows. In Section 2, we discuss the Dirac equations in the spacetime. In Section 3, we investigate Hawking radiation using DamourRuffini method. In Section 4, when energy conservation and particles' back reaction to the spacetime are taken into account, we obtain the emission spectrum which is no longer thermal. At last, we finish this paper with some conclusions. 


\section{Dirac Equations in the Static Riemann Black Hole}

The metric of the static Riemann spacetime can be expressed as

$$
d s^{2}=a^{2} d t^{2}-b^{2} d x^{2}-c^{2} d y^{2}-d^{2} d z^{2}
$$

where $a, b, c, d$ are the functions of $(x, y, x)$ and the position of event horizon is given by $x=\xi$.

It is very convenient to study Dirac equations in curved background using Newman-Penrose formalism [21, 22]. From metric (1), we choose a set of null tetrad frame as

$$
\begin{aligned}
l^{\mu} & =\frac{1}{\sqrt{2}}\left(\frac{1}{a},-\frac{1}{b}, 0,0\right), \\
n^{\mu} & =\frac{1}{\sqrt{2}}\left(\frac{1}{a}, \frac{1}{b}, 0,0\right), \\
m^{\mu} & =\frac{1}{\sqrt{2}}\left(0,0,-\frac{1}{c},-\frac{i}{d}\right), \\
\bar{m}^{\mu} & =\frac{1}{\sqrt{2}}\left(0,0,-\frac{1}{c}, \frac{i}{d}\right),
\end{aligned}
$$

and the corresponding dual forms are

$$
\begin{aligned}
l_{\mu} & =\frac{1}{\sqrt{2}}(a, b, 0,0), \\
n_{\mu} & =\frac{1}{\sqrt{2}}(a,-b, 0,0), \\
m_{\mu} & =\frac{1}{\sqrt{2}}(0,0, c, i d), \\
\bar{m}_{\mu} & =\frac{1}{\sqrt{2}}(0,0, c,-i d),
\end{aligned}
$$

where $l^{\mu} n_{\mu}=-1, m^{\mu} \bar{m}_{\mu}=1$ and $l^{\mu} m_{\mu}=0, n^{\mu} m_{\mu}=0$.

So we can calculate 12 Newman-Penrose coefficients [22, 23] from (1), (2), and (3):

$$
\begin{gathered}
\kappa=\frac{1}{2 \sqrt{2}}\left(\frac{1}{a c} \frac{\partial}{\partial y} a-\frac{1}{b c} \frac{\partial}{\partial y} b+\frac{i}{a d} \frac{\partial}{\partial z} a-\frac{i}{b d} \frac{\partial}{\partial z} b\right) \\
\pi=-\frac{1}{2 \sqrt{2}}\left(\frac{1}{a c} \frac{\partial}{\partial y} a+\frac{1}{b c} \frac{\partial}{\partial y} b-\frac{i}{a d} \frac{\partial}{\partial z} a-\frac{i}{b d} \frac{\partial}{\partial z} b\right) \\
\varepsilon=-\frac{1}{2 \sqrt{2}} \frac{1}{a b} \frac{\partial}{\partial x} a \\
\rho=\frac{1}{2 \sqrt{2}}\left(\frac{1}{b c} \frac{\partial}{\partial x} c+\frac{1}{b d} \frac{\partial}{\partial x} d\right) \\
\lambda=\frac{1}{2 \sqrt{2}}\left(\frac{1}{b c} \frac{\partial}{\partial x} c-\frac{1}{b d} \frac{\partial}{\partial x} d\right) \\
\alpha=\frac{1}{2 \sqrt{2}}\left(\frac{1}{c d} \frac{\partial}{\partial y} d-\frac{i}{c d} \frac{\partial}{\partial z} c\right)
\end{gathered}
$$

$$
\begin{gathered}
\sigma=\lambda, \\
\mu=\rho, \\
\beta=-\bar{\alpha}, \\
\nu=-\bar{\kappa}, \\
\tau=-\bar{\pi}, \\
\gamma=\varepsilon .
\end{gathered}
$$

In the curved spacetime, Dirac equations of a particle in Newman-Penrose formalism are given as [24]

$$
\begin{gathered}
(D+\varepsilon-\rho) F_{1}+(\bar{\delta}+\pi-\alpha) F_{2}=\frac{i \mu}{\sqrt{2}} G_{1}, \\
(\delta+\beta-\tau) F_{1}+(\Delta+\mu-\gamma) F_{2}=\frac{i \mu}{\sqrt{2}} G_{2}, \\
-(\delta+\bar{\pi}-\bar{\alpha}) G_{1}+(D+\bar{\varepsilon}-\bar{\rho}) G_{2}=\frac{i \mu}{\sqrt{2}} F_{2}, \\
(\Delta+\bar{\mu}-\bar{\gamma}) G_{1}-(\bar{\delta}+\bar{\beta}-\bar{\tau}) G_{2}=\frac{i \mu}{\sqrt{2}} F_{1},
\end{gathered}
$$

where $F_{1}, F_{2}, G_{1}, G_{2}$ are the four components of the wave function $\psi ; \mu$ is rest mass; and $D, \Delta, \delta, \bar{\delta}$ are defined by

$$
D=l^{\mu} \partial_{\mu}, \quad \Delta=n^{\mu} \partial_{\mu}, \quad \delta=m^{\mu} \partial_{\mu}, \quad \bar{\delta}=\bar{m}^{\mu} \partial_{\mu} .
$$

By putting NP coefficients (4) into (5), we get the equations in an explicit form:

$$
\begin{aligned}
& {\left[\frac{1}{a} \frac{\partial}{\partial t}-\frac{1}{b} \frac{\partial}{\partial x}-\frac{1}{2 b}\left(\frac{\partial}{\partial x} \ln a c d\right)\right] F_{1}} \\
& +\left[-\frac{1}{c} \frac{\partial}{\partial y}+\frac{i}{d} \frac{\partial}{\partial z}-\frac{1}{2 c}\left(\frac{\partial}{\partial y} \ln a b d\right)+\frac{i}{2 d}\left(\frac{\partial}{\partial z} \ln a b c\right)\right] F_{2} \\
& -i \mu G_{1}=0, \\
& {\left[\frac{1}{a} \frac{\partial}{\partial t}+\frac{1}{b} \frac{\partial}{\partial x}+\frac{1}{2 b}\left(\frac{\partial}{\partial x} \ln a c d\right)\right] F_{2}} \\
& +\left[-\frac{1}{c} \frac{\partial}{\partial y}-\frac{i}{d} \frac{\partial}{\partial z}-\frac{1}{2 c}\left(\frac{\partial}{\partial y} \ln a b d\right)-\frac{i}{2 d}\left(\frac{\partial}{\partial z} \ln a b c\right)\right] F_{1} \\
& -i \mu G_{2}=0, \\
& {\left[\frac{1}{a} \frac{\partial}{\partial t}+\frac{1}{b} \frac{\partial}{\partial x}+\frac{1}{2 b}\left(\frac{\partial}{\partial x} \ln a c d\right)\right] G_{1}} \\
& +\left[\frac{1}{c} \frac{\partial}{\partial y}-\frac{i}{d} \frac{\partial}{\partial z}+\frac{1}{2 c}\left(\frac{\partial}{\partial y} \ln a b d\right)-\frac{i}{2 d}\left(\frac{\partial}{\partial z} \ln a b c\right)\right] G_{2} \\
& -i \mu F_{1}=0, \\
& +\left[\frac{1}{c} \frac{\partial}{\partial y}+\frac{i}{d} \frac{\partial}{\partial z}+\frac{1}{2 c}\left(\frac{\partial}{\partial y} \ln a b d\right)+\frac{i}{2 d}\left(\frac{\partial}{\partial z} \ln a b c\right)\right] G_{1} \\
& {\left[\frac{1}{a} \frac{\partial}{\partial t}-\frac{1}{b} \frac{\partial}{\partial x}-\frac{1}{2 b}\left(\frac{\partial}{\partial x} \ln a c d\right)\right] G_{2}} \\
& \left.+\frac{1}{\partial x}\right)
\end{aligned}
$$


and these equations could also be rewritten in a more concise matrix form:

$$
\begin{aligned}
& \left\{\gamma^{0} \frac{\partial}{\partial t}+\gamma^{1}\left[\frac{\partial}{\partial x}+\frac{1}{2}\left(\frac{\partial}{\partial x} \ln a c d\right)\right]\right. \\
& +\gamma^{2}\left[\frac{\partial}{\partial y}+\frac{1}{2}\left(\frac{\partial}{\partial y} \ln a b d\right)\right]+\gamma^{3}\left[\frac{\partial}{\partial z}+\frac{1}{2}\left(\frac{\partial}{\partial z} \ln a b c\right)\right] \\
& -i \mu I\} \psi=0
\end{aligned}
$$

where

$$
\begin{gathered}
\psi=\left(F_{1}, F_{2}, G_{1}, G_{2}\right)^{T}, \\
\gamma^{0} \equiv \frac{1}{a}\left(\begin{array}{cccc}
0 & 0 & 1 & 0 \\
0 & 0 & 0 & 1 \\
1 & 0 & 0 & 0 \\
0 & 1 & 0 & 0
\end{array}\right) \equiv \frac{1}{a} \widehat{\gamma}^{0}, \\
\gamma^{1} \equiv \frac{1}{b}\left(\begin{array}{cccc}
0 & 0 & 1 & 0 \\
0 & 0 & 0 & -1 \\
-1 & 0 & 0 & 0 \\
0 & 1 & 0 & 0
\end{array}\right) \equiv \frac{1}{b} \widehat{\gamma}^{1}, \\
\gamma^{2} \equiv \frac{1}{c}\left(\begin{array}{cccc}
0 & 0 & 0 & 1 \\
0 & 0 & 1 & 0 \\
0 & -1 & 0 & 0 \\
-1 & 0 & 0 & 0
\end{array}\right) \equiv \frac{1}{c} \widehat{\gamma}^{2}, \\
\gamma^{3} \equiv \frac{1}{d}\left(\begin{array}{cccc}
0 & 0 & 0 & -i \\
0 & 0 & i & 0 \\
0 & i & 0 & 0 \\
-i & 0 & 0 & 0
\end{array}\right) \equiv \frac{1}{d} \widehat{\gamma}^{3}, \\
\gamma^{\mu} \gamma^{\nu}+\gamma^{\nu} \gamma^{\mu}=2 g^{\mu \nu} I,
\end{gathered}
$$

in which $\gamma^{\mu}$ is the $\gamma$ metric in curved spacetime and $I$ is the identity matrix.

Defining another variable $\widehat{\psi}$ as

$$
\psi=\frac{1}{\sqrt{a c d}} \widehat{\psi}
$$

and putting (10) into (8), we have

$$
\begin{aligned}
& \left\{\gamma^{0} \frac{\partial}{\partial t}+\gamma^{1} \frac{\partial}{\partial x}+\gamma^{2}\left[\frac{\partial}{\partial y}+\frac{1}{2}\left(\frac{\partial}{\partial y} \ln \frac{b}{c}\right)\right]\right. \\
& \left.+\gamma^{3}\left[\frac{\partial}{\partial z}+\frac{1}{2}\left(\frac{\partial}{\partial z} \ln \frac{b}{d}\right)\right]-i \mu I\right\} \widehat{\psi}=0 .
\end{aligned}
$$

So we get the Dirac equations in a very simple and concise form, which is helpful for the following disccusion.

\section{Analytic Extension and Hawking Thermal Radiation}

We could always write [25]

$$
\begin{aligned}
g_{00}=q^{2}(x, y, z)(x-\xi)^{n}, & g^{11}=-p^{2}(x, y, z)(x-\xi)^{m}, \\
g^{22}=\frac{\theta}{(x-\xi)^{q}}, & g^{33}=\frac{\varphi}{(x-\xi)^{r}}
\end{aligned}
$$

where $x=\xi$ is the position of event horizon and $q^{2}, p^{2}, \theta, \varphi$ are arbitary fuctions which are not zero and nonsingular at the horizon.

The surface gravity $\kappa$ is given by [25]

$$
\begin{aligned}
\kappa=\frac{1}{2} \lim _{x \rightarrow \xi} \sqrt{-\frac{g^{11}}{g_{00}}} \frac{\partial}{\partial x} g_{00} \\
=\lim _{x \rightarrow \xi}\left\{\frac{1}{2} n p q(x-\xi)^{(1 / 2)(m+n-2)}\right. \\
\left.\times\left[1+\frac{2}{n}\left(\frac{\partial}{\partial x} \ln q\right)(x-\xi)\right]\right\} .
\end{aligned}
$$

Because $\kappa$ is not zero and non singular, we need that

$$
m+n=2, \quad n \neq 0 .
$$

Another consideration is the area of event horizon

$$
\begin{aligned}
A & =\int d A=\int\left|\begin{array}{cc}
g_{22} & 0 \\
0 & g_{33}
\end{array}\right|^{1 / 2} d y d z=\int \sqrt{g_{22} g_{33}} d y d z \\
& =\int \sqrt{\frac{1}{\theta \varphi}}(x-\xi)^{(1 / 2)(q+r)} d z d y .
\end{aligned}
$$

It is well known that the area of the event horizon is not zero and non singular, so we must require that

$$
q+r=0
$$

At last, because the determinant of the metric is also not zero and nonsingular at the horizon, it is easy to obtain that

$$
n-m=0 .
$$

From (14) and (17), we obtain

$$
m=n=1 .
$$

so the surface gravity is

$$
\kappa=\frac{1}{2} p(\xi) q(\xi) .
$$

Tortoise coordinate transformation can be given as

$$
\widehat{x}=\frac{1}{2 \kappa} \ln \frac{x-\xi}{\xi} .
$$


Then, we have

$$
\begin{aligned}
d \widehat{x} & =\frac{1}{2 \kappa(x-\xi)} d x, \\
\frac{\partial}{\partial x} & =\frac{1}{2 \kappa(x-\xi)} \frac{\partial}{\partial \widehat{x}} .
\end{aligned}
$$

By putting (22) into (11), we get

$$
\begin{aligned}
& {\left[\widehat{\gamma}^{0} \frac{1}{q(x-\xi)^{1 / 2}} \frac{\partial}{\partial t}+\widehat{\gamma}^{1} \frac{1}{q}(x-\xi)^{-1 / 2} \frac{\partial}{\partial \widehat{x}}+\widehat{r}^{2} \frac{1}{c} \frac{\partial}{\partial y}\right.} \\
& -\widehat{r}^{2} \frac{1}{2 c}\left(\frac{\partial}{\partial y} \ln p c\right)+\widehat{\gamma}^{3} \frac{1}{d} \frac{\partial}{\partial z}-\widehat{\gamma}^{3} \frac{1}{2 d}\left(\frac{\partial}{\partial z} \ln p d\right) \\
& -i \mu I] \widehat{\psi}=0 .
\end{aligned}
$$

Multiplying (23) by $q(x-\xi)^{1 / 2}$ and considering that $(x-$ $\xi) \ll 1$ near the horizon, we can obtain the equation near the event horizon

$$
\left(\widehat{\gamma}^{0} \frac{\partial}{\partial t}+\widehat{\gamma}^{1} \frac{\partial}{\partial \widehat{x}}\right) \widehat{\psi}=0
$$

Because $(\partial / \partial t)^{a}$ is a Killing vector field, we can separate variable as

$$
\widehat{\psi}=e^{-i \omega t} \phi(\widehat{x}) \chi(y, z),
$$

where $\omega$ is the energy of radiating particle, and we get the radial wave equation which we are interested in:

$$
-i \widehat{\gamma}^{0} \omega \phi(\widehat{x})+\widehat{\gamma}^{1} \frac{d}{d \widehat{x}} \phi(\widehat{x})=0
$$

where

$$
\phi(\widehat{x})=\left(f_{1}, f_{2}, g_{1}, g_{2}\right)^{T} .
$$

The solutions are

$$
\begin{aligned}
& f_{1}=e^{-i \omega \hat{x}}, \\
& f_{2}=e^{i \omega \hat{x}}, \\
& g_{1}=e^{i \omega \hat{x}}, \\
& g_{2}=e^{-i \omega \hat{x}} .
\end{aligned}
$$

So it is easy to show that just outside the horizon $(x>\xi)$ two linearly independent solutions exist: the ingoing wave function is

$$
\psi_{\omega}^{\text {in }} \sim e^{-i w(t+\widehat{x})} \frac{1}{\sqrt{2}}(1,0,0,1)^{T}=e^{-i \omega V} \frac{1}{\sqrt{2}}(1,0,0,1)^{T},
$$

and the outgoing wave function is

$$
\begin{aligned}
\psi_{\omega}^{\text {out }} & \sim e^{-i \omega(t-\widehat{x})} \frac{1}{\sqrt{2}}(0,1,1,0)^{T}=e^{-i \omega V} e^{2 i \omega \widehat{x}} \frac{1}{\sqrt{2}}(0,1,1,0)^{T} \\
& =e^{-i \omega V}\left(\frac{x-\xi}{\xi}\right)^{i \omega / \kappa} \frac{1}{\sqrt{2}}(0,1,1,0)^{T},
\end{aligned}
$$

where we use the usual advanced Eddington-Finkelstein coordinate, $V=t+\widehat{x}$.

We are concerned about the radiation of black hole, so we only need to consider (30), while this equation is not analytic on the horizon and therefore cannot be straightforwardly extended to the region inside the horizon. Following Damour and Ruffini's method [26], we can extend the outgoing wave equation (30) inside the horizon by turning the $(-\pi)$ angle through the negative half complex plane. Let

$$
(x-\xi) \longrightarrow|x-\xi| e^{-i \pi}=(\xi-x) e^{-i \pi},
$$

and the outgoing wave function inside the horizon is

$$
\begin{aligned}
\psi_{\omega}^{\text {out }}(x<\xi) & =e^{-i \omega V}\left[\frac{\xi-x}{\xi} e^{-i \pi}\right]^{i \omega / \kappa} \frac{1}{\sqrt{2}}(0,1,1,0)^{T} \\
& =e^{-i \omega V} e^{\pi \omega / \kappa}\left(\frac{\xi-x}{\xi}\right)^{i \omega / \kappa} \frac{1}{\sqrt{2}}(0,1,1,0)^{T} \\
& =e^{-i \omega V} e^{\pi \omega / \kappa} e^{2 i \omega(1 / 2 \kappa) \ln ((\xi-x) / \xi)} \frac{1}{\sqrt{2}}(0,1,1,0)^{T} \\
& =e^{-i \omega V} e^{\pi \omega / \kappa} e^{2 i \omega \widehat{x}} \frac{1}{\sqrt{2}}(0,1,1,0)^{T}
\end{aligned}
$$

Please notice that $\widehat{x}=(1 / 2 \kappa) \ln ((\xi-x) / \xi)$ is the tortoise coordinate transformation inside the horizon.

Therefore, according to Sannan's work [27], the emission rate at the horizon is

$$
\Gamma=\left|\frac{\psi_{\omega}^{\text {out }}(x>\xi)}{\psi_{\omega}^{\text {out }}(x<\xi)}\right|^{2}=e^{-2 \pi \omega / \kappa} .
$$

Then, it is easy to obtain the thermal radiation spectrum

$$
N_{w}=\frac{1}{e^{2 \pi \omega / \kappa}+1}=\frac{1}{e^{\omega / T}+1},
$$

where $T=\kappa / 2 \pi$ is the temperature of the horizon and $\kappa$ is the surface gravity.

\section{Back Reaction to the Spacetime}

In Section 3, we obtain Hawking's purely thermal spectrum equations (33) and (34), without considering the back reaction of emitting particles to the spacetime, which will lead to the information loss puzzle. As a matter of fact, when a particle with energy $\omega_{i}$ radiates from the black hole whose mass is $M$, the mass of the black hole $M$ should reduce to $M-\omega_{i}$, and the emission rate should be

$$
\Gamma_{i}=e^{-2 \pi \omega_{i} / \kappa_{i}},
$$

where $\kappa_{i}$ is the surface gravity of the black hole after emitting this particle.

For many particles' emission, assuming that they radiate one by one, we have

$$
\Gamma=\prod_{i} \Gamma_{i}=e^{\sum_{i}\left(-2 \pi \omega_{i} / \kappa_{i}\right)} .
$$


And we could regard the emission as a continuous process, so the sum in (36) should be replaced by integration

$$
\Gamma=e^{\int_{0}^{w}-(d \omega / T)}=e^{\int_{M}^{M-\omega}(d M / T)}=e^{S_{2}-S_{1}}=e^{\Delta S},
$$

where $\Delta S$ is the difference between the entropy of the black hole before and after emission, and note that we have used the first law of black hole thermodynamics $d S=d M / T$, which manifests that Hawking radiation should be a quasistatic process.

So we obtain the modified Hawking radiation, which is consistent with the tunneling method of Parikh. We find that Hawking radiation is not a pure thermal spectrum, the deviation from pure thermal spectrum could explain the information loss paradox, and the result is precisely consistent with an underlying unitary theory. According to Parikh's discussion [11, 12], "Quantum mechanics tells us that the rate must be expressed as

$$
\Gamma(i \longrightarrow f)=\left|M_{f i}\right|^{2} \cdot(\text { phase space factor }),
$$

where the first term on the right is the square of the amplitude for the process. The phase space factor is obtained by summing over final states and averaging over initial states. But the number of final states is just the final exponent of the final entropy, while the number of initial states is the exponent of the initial entropy." So, we have

$$
\Gamma \sim \frac{e^{S_{\text {final }}}}{e^{S_{\text {initial }}}}=\exp (\Delta S),
$$

which is in agreement with our resultant equation (37). This suggests that the formula we have is actually exact, up to a prefactor.

Next we will give a concrete example [28], Schwarzschild Black Hole, and we would see more clearly.

Following the same step above, we could first get Hawking's thermal spectrum of Dirac particles:

$$
\Gamma=e^{-8 \pi M w}=e^{-w / T},
$$

where $T=1 / 8 \pi M$ is the Hawking temperature.

If we consider back reaction, the emission rate is

$$
\Gamma=\prod_{i} \Gamma_{i}=e^{-8 \pi \int_{0}^{w}\left(M-w^{\prime}\right) d w^{\prime}}=e^{-8 \pi M w(1-(w / 2 M))},
$$

and we can calculate the difference of the entropy before and after emission easily:

$$
\begin{aligned}
\Delta S_{\mathrm{BH}} & =\frac{\Delta A}{4}=\frac{1}{4}\left[4 \pi(2(M-w))^{2}-4 \pi(2 M)^{2}\right] \\
& =-8 \pi M w\left(1-\frac{w}{2 M}\right),
\end{aligned}
$$

so we also get $\Gamma=e^{\Delta S_{\mathrm{BH}}}$, which coincides with the above general discussion. We find that when we neglect the quadratic term in the exponential part, (41) becomes a Boltzmann factor, and otherwise, the spectrum is not purely thermal, which would explain the information puzzle, and some information could be taken out from black hole in terms of the higher term.

\section{Discussion and Conclusion}

This paper carefully investigates Hawking radiation (37) of massive Dirac particles from a general static Riemann spacetime using improved Damour-Ruffini's method. In 1983, [23] proved that Dirac particles radiate thermally in this spacetime. In our work, we consider energy conservation and the back reaction of radiating particles to the spacetime and have concluded that the radiation spectrum cannot be strictly thermal, and information can be taken out from the black hole. Our result is the same as Parikh and Wilczek's tunneling method and is also consistent with an underlying unitary theory. Because our metric is general, not only to get the corrected spectrum using Parikh and Wilczek's tunneling method is not easy, but also the theory of this paper is general, and we use this improved Damour-Ruffini's method to get the important result.

Actually, by the method of classical Damour-Ruffini method, without considering the back reaction to the spacetime, we could get the blackbody spectrum near the black hole. But the equation of emergent wave should contain a potential barrier between the horizon and the infinity, which characterizes the spacetime curvature outside the black hole, so the observer at infinity could see a greybody spectrum. The blackbody spectrum near the horizon is scattered by the potential barrier and becomes the greybody spectrum at infinity. And we often call Hawking radiation blackbody spectrum, which implies the spectrum near the horizon.

\section{Acknowledgments}

The authors are grateful for Professor Rong-Gen Cai and Professor Zheng Zhao for their useful discussions and comments. The authors would also like to thank the referee of this paper for the comments which are very helpful for them to improve the work. The work is supported by the Project of Knowledge Innovation Program of Chinese Academy of Sciences (no. KJCX2.YW.W10) and the National Natural Science Foundation of China (no. 11275017 and no. 11173028).

\section{References}

[1] S. W. Hawking, "Black hole explosions?" Nature, vol. 248, no. 5443, pp. 30-31, 1974.

[2] S. W. Hawking, "Particle creation by black holes," Communications in Mathematical Physics, vol. 43, no. 3, pp. 199-220, 1975.

[3] J. D. Bekenstein, "Black holes and entropy," Physical Review. D, vol. 7, pp. 2333-2346, 1973.

[4] J. M. Bardeen, B. Carter, and S. W. Hawking, "The four laws of black hole mechanics," Communications in Mathematical Physics, vol. 31, pp. 161-170, 1973.

[5] S. W. Hawking, "Breakdown of predictability in gravitational collapse," Physical Review D, vol. 14, no. 10, pp. 2460-2473, 1976.

[6] S. W. Hawking, "Information loss in black holes," Physical Review D, vol. 72, no. 8, Article ID 084013, 4 pages, 2005.

[7] J. Preskill, "Do black holes destroy information," in Proceedings of the International Symposium on Black Holes, Membranes, Wormholes, and Superstrings, The Woodlands, Tex, USA, January 1992. 
[8] C. G. Callan Jr. and J. M. Maldacena, "D-brane approach to black hole quantum mechanics," Nuclear Physics B, vol. 472, no. 3, pp. 591-608, 1996.

[9] S. D. Mathur, "The information paradox: a pedagogical introduction," Classical and Quantum Gravity, vol. 26, no. 22, Article ID 224001, 2009.

[10] M. K. Parikh and F. Wilczek, "Hawking radiation as tunneling," Physical Review Letters, vol. 85, no. 24, pp. 5042-5045, 2000.

[11] M. K. Parikh, "Energy conservation and Hawking radiation," in Proceedings of the 10th Marcel Grossmann Meeting, Rio de Janeiro, Brazil, July 2003.

[12] M. Parikh, "A secret tunnel through the horizon," International Journal of Modern Physics D, vol. 13, no. 10, pp. 2351-2354, 2004.

[13] J.-Y. Zhang and Z. Zhao, "Charged particles' tunnelling from the Kerr-Newman black hole," Physics Letters B, vol. 638, no. 2-3, pp. 110-113, 2006.

[14] J.-Y. Zhang and Z. Zhao, "Hawking radiation of charged particles via tunneling from the Reissner-Nordström black hole," Journal of High Energy Physics A, vol. 2005, no. 10, 2005.

[15] J.-Y. Zhang and Z. Zhao, "New coordinates for Kerr-Newman black hole radiation," Physics Letters B, vol. 618, no. 1-4, pp. 1422, 2005.

[16] J.-Y. Zhang and Z. Zhao, "Hawking radiation via tunneling from Kerr black holes," Modern Physics Letters A, vol. 20, no. 22, pp. 1673-1681, 2005.

[17] J.-Y. Zhang and Z. Zhao, "Massive particles' black hole tunneling and de Sitter tunneling," Nuclear Physics B, vol. 725, no. 1-2, pp. 173-180, 2005.

[18] W.-B. Liu, "Damour-Ruffini method and nonthermal Hawking radiation from the Reissner-Nordstrom black hole," Acta Physica Sinica, vol. 56, no. 10, pp. 6164-6168, 2007.

[19] X. He and W. Liu, "Modified Hawking radiation in a BTZ black hole using Damour-Ruffini method," Physics Letters B, vol. 653, no. 2-4, pp. 330-334, 2007.

[20] S. Zhou and W. Liu, "Hawking radiation of charged Dirac particles from a Kerr-Newman black hole," Physical Review D, vol. 77, no. 10, Article ID 104021, 6 pages, 2008.

[21] R. Penrose and W. Rindler, Spinors and Space-Time, Cambridge Monographs on Mathematical Physics, Cambridge University Press, Cambridge, UK, 1984.

[22] S. Chandrasekhar, The Mathematical Theory of Black Holes, vol. 69 of International Series of Monographs on Physics, Oxford University Press, New York, NY, USA, 1983.

[23] D.Y. Xu, "The Hawking radiation of Dirac particles in a 4Ddimensional stationary Riemannian space-time," Chinese Science Bulletin, vol. 28, no. 11, pp. 650-654, 1983 (Chinese).

[24] D. N. Page, "Dirac equation around a charged, rotating black hole," Physical Review D, vol. 14, no. 6, pp. 1509-1510, 1976.

[25] Z. Zhao, Thermal Nature of Black Holes and the Singularity of the Spacetime, Beijing Normal University Press, Beijing, China, 1999.

[26] T. Damour and R. Ruffini, "Black-hole evaporation in the KleinSauter-Heisenberg-Euler formalism," Physical Review D, vol. 14, no. 2, pp. 332-334, 1976.

[27] S. Sannan, "Heuristic derivation of the probability distributions of particles emitted by a black hole," General Relativity and Gravitation, vol. 20, no. 3, pp. 239-246, 1988.

[28] X. He and W. Liu, "Dirac particles' hawking radiation from a Schwarzschild black hole," Chinese Physics Letters, vol. 24, no. 8, article 080, pp. 2448-2450, 2007. 

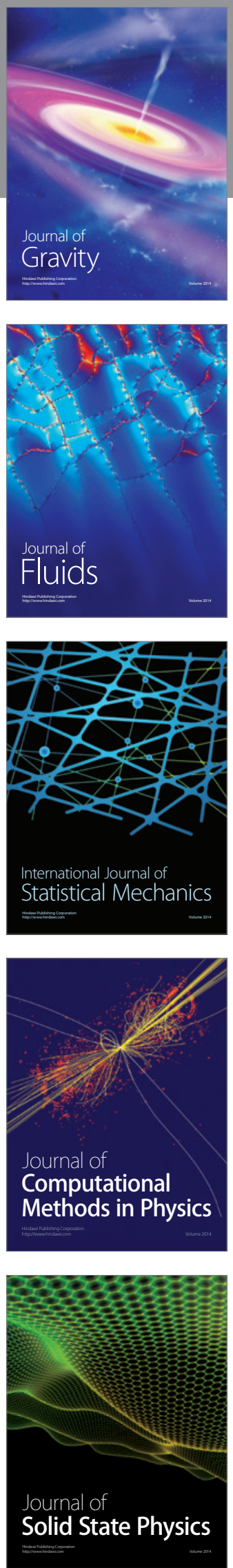

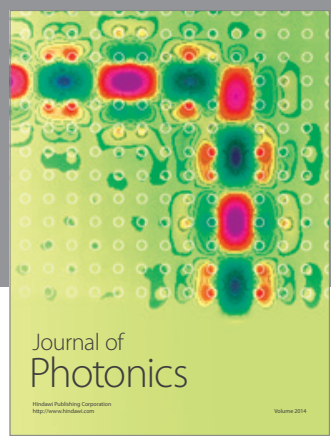

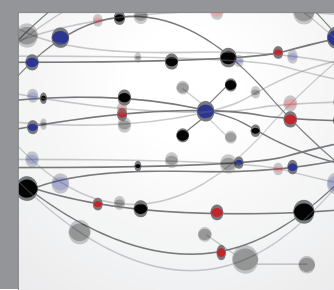

The Scientific World Journal

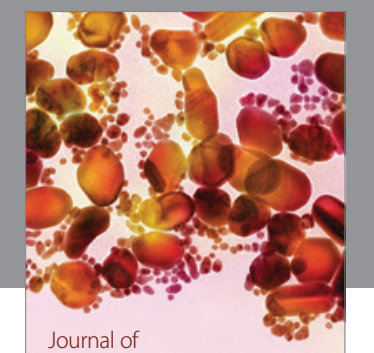

Soft Matter
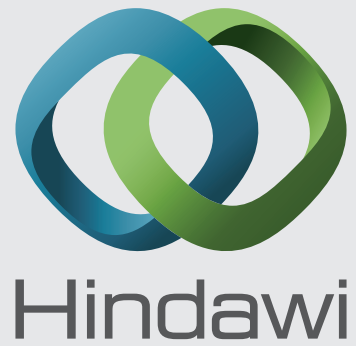

Submit your manuscripts at

http://www.hindawi.com
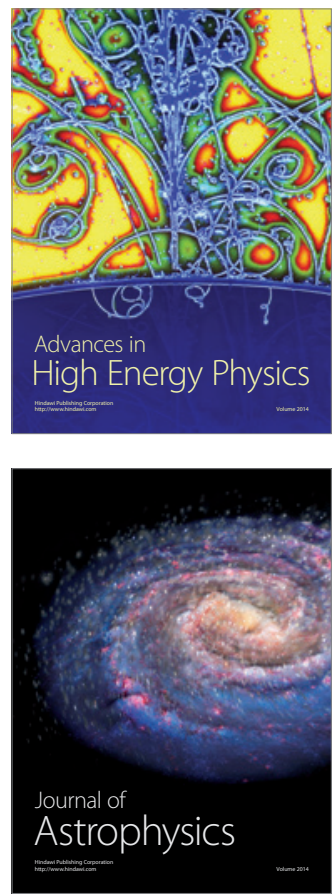
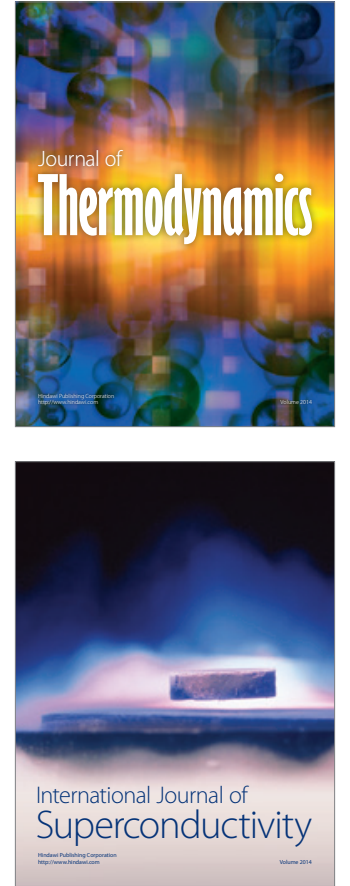
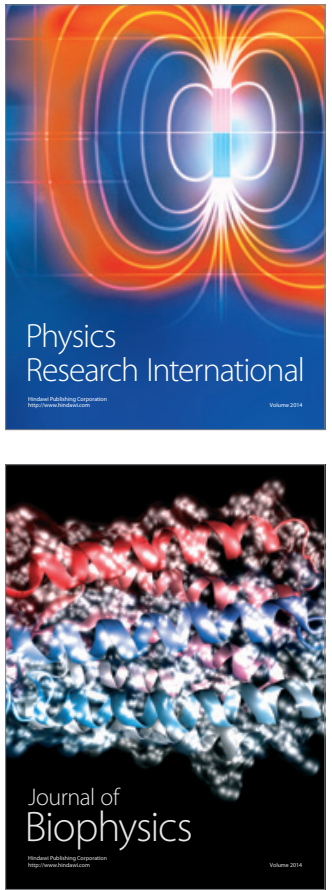
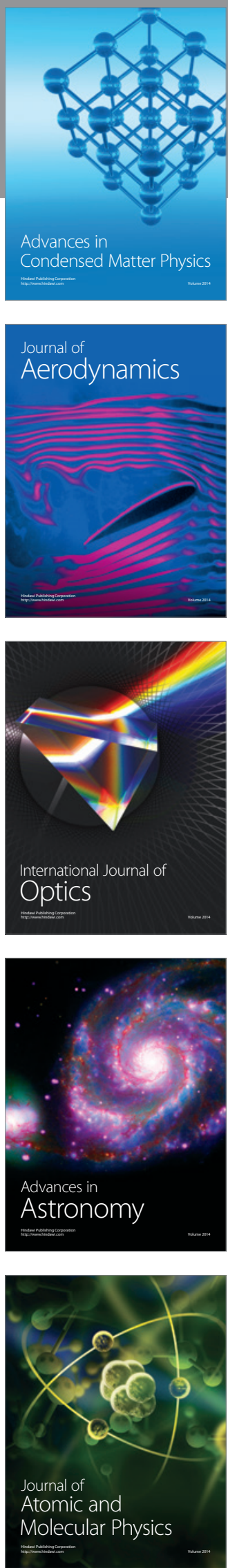